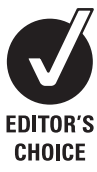

See Editorial, p 847

- Supplementary tables 1-4 and supplementary fig 1 are published online only at http:// thorax.bmj.com/content/vol63/ issue10

${ }^{1}$ Department of Respiratory Medicine, City Hospital, Birmingham, UK; ${ }^{2}$ Lung Injury and Fibrosis Treatment Programme, Division of Medical Sciences, University of Birmingham, UK; ${ }^{3}$ Division of Immunity and Infection, University of Birmingham, UK;

${ }^{4}$ Department of Respiratory Medicine, Heartlands Hospital, Birmingham, UK; ${ }^{5}$ Department of Respiratory Medicine, University Hospital, Birmingham, UK

Correspondence to: Dr N Nathani, Department of Thoracic Medicine, City Hospital, Dudley Road, Birmingham B18 70H, UK; n.nathani@nhs.net

Received 26 November 2007 Accepted 28 March 2008 Published Online First 20 May 2008

\title{
Churg-Strauss syndrome and leukotriene antagonist use: a respiratory perspective
}

\author{
N Nathani, ${ }^{1}, 2$ M A Little, ${ }^{3}$ H Kunst, ${ }^{4}$ D Wilson, ${ }^{5}$ D R Thickett ${ }^{2}$
}

\begin{abstract}
Background: Churg-Strauss syndrome (CSS) is a rare granulomatous small vessel vasculitis that occurs against a background of longstanding asthma. Leukotriene antagonists (LTAs) are used in the management of asthma and may facilitate a reduction in steroid dosage. Reports of the development of CSS in patients with asthma following the initiation of LTA therapy suggest either a causal association or an unmasking of latent CSS as steroid doses fall. We have undertaken a systematic review to establish whether evidence of a drug induced syndrome exists.
\end{abstract}

Methods: Systematic review searching Medline from database inception to August 2007 to identify cases with a possible association between LTAs and CSS. Hill's criteria of causation were used to assess strength of causality.

Results: 62 cases in which CSS developed after the introduction of LTA therapy were identified. Patients were divided into three groups: group 1 had received no previous steroid therapy; group 2 had been treated with oral and/or inhaled corticosteroids, but had no change in steroid therapy following LTA introduction; and group 3 had a clear reduction in steroid therapy following introduction of LTA therapy. The majority of patients from each group exhibited a clear temporal relationship between initiation of LTA and development of CSS, with no evidence of pre-existing disease.

Conclusions: Currently available evidence suggests an association between LTA and CSS that may be causal.

Churg-Strauss syndrome (CSS) is an uncommon disease with an unclear cause and an estimated annual incidence of 2.7 per million patient years. ${ }^{1}$ In 1990, the American College of Rheumatology (ACR) suggested that patients should have four out of six criteria (asthma, eosinophilia $>10 \%$, mononeuropathy or polyneuropathy, non-fixed pulmonary infiltrates, paranasal sinus abnormality and extravascular eosinophilia) for diagnosis of $\mathrm{CSS}^{2}$ (see supplementary table 1 online).

CSS has characteristic histological findings and the association with asthma distinguishes it from other vasculitides. It has been recognised that, in many but not all patients, the disease follows a characteristic three stage course ${ }^{3}$ (see supplementary fig 1 online). Asthma and/or allergic rhinitis, with or without nasal polyposis, precedes full development of the syndrome, often by many years. Differentiating CSS from other disorders with eosinophilic tissue infiltration is difficult and, in the absence of histopathologically demonstrable small vessel vasculitis, the clinical context (particularly the presence of a history of asthma) is highly relevant for a diagnosis of CSS.
Antineutrophil cytoplasmic antibodies (ANCA) are not generally viewed as the primary cause of the disease as up to $50 \%$ of patients with CSS do not express these antibodies. ${ }^{45}$

Leukotriene antagonists (LTAs) are a relatively recent addition to the treatment options in asthma. These drugs include one enzyme inhibitor of 5-lipoxygenase (Zileuton) and chemically distinct cysteinyl leukotriene type I (cys-LT1) receptor antagonists such as zafirlukast, pranlukast and montelukast. These agents neutralise the effects of the cysteinyl leukotrienes (LTC4, LTD4 and LTE4) by selectively antagonising cys-LT1 receptors.

A number of case series and reports in the recent literature suggest an association between LTAs and CSS with a number of patients developing CSS following the introduction of LTA therapy. The low incidence of CSS following treatment with LTAs makes investigation of this issue with controlled studies of sufficient power difficult and to date there are no systematic reviews collating this evidence. We have therefore undertaken a systematic review to explore further the possibility of a causal association.

\section{METHOD}

\section{Identification of potential studies}

We conducted a systematic literature review to identify studies that reported an association between CSS and LTA use. Studies were identified using MEDLINE (1950 to August 2007), limiting study selection to citations in the English language. The search term combinations used were: leukotriene antagonists and Churg-Strauss syndrome, montelukast and Churg-Strauss syndrome, zafirlukast and Churg-Strauss syndrome, pranlukast and Churg-Strauss Syndrome, zileuton and Churg-Strauss syndrome. The search revealed 212 citations that underwent subsequent review (see fig 1).

\section{Assessment of CSS and LTA use}

Data were collected on age and gender of the patients, history of asthma, present and past treatment, nature of corticosteroid treatment, type of LTA used, duration of LTA therapy prior to the development of CSS, organ involvement and ANCA status. The presence of dormant, suppressed or forme fruste disease ${ }^{6}$ before the introduction of LTA therapy was assessed according to predetermined criteria (see supplementary table 1 online). Where possible, the duration, route and timing of steroid therapy in relation to LTA introduction were recorded. 
Figure 1 Summary of studies assessed and included in this review. CSS, ChurgStrauss syndrome; LTA, leukotriene antagonists.

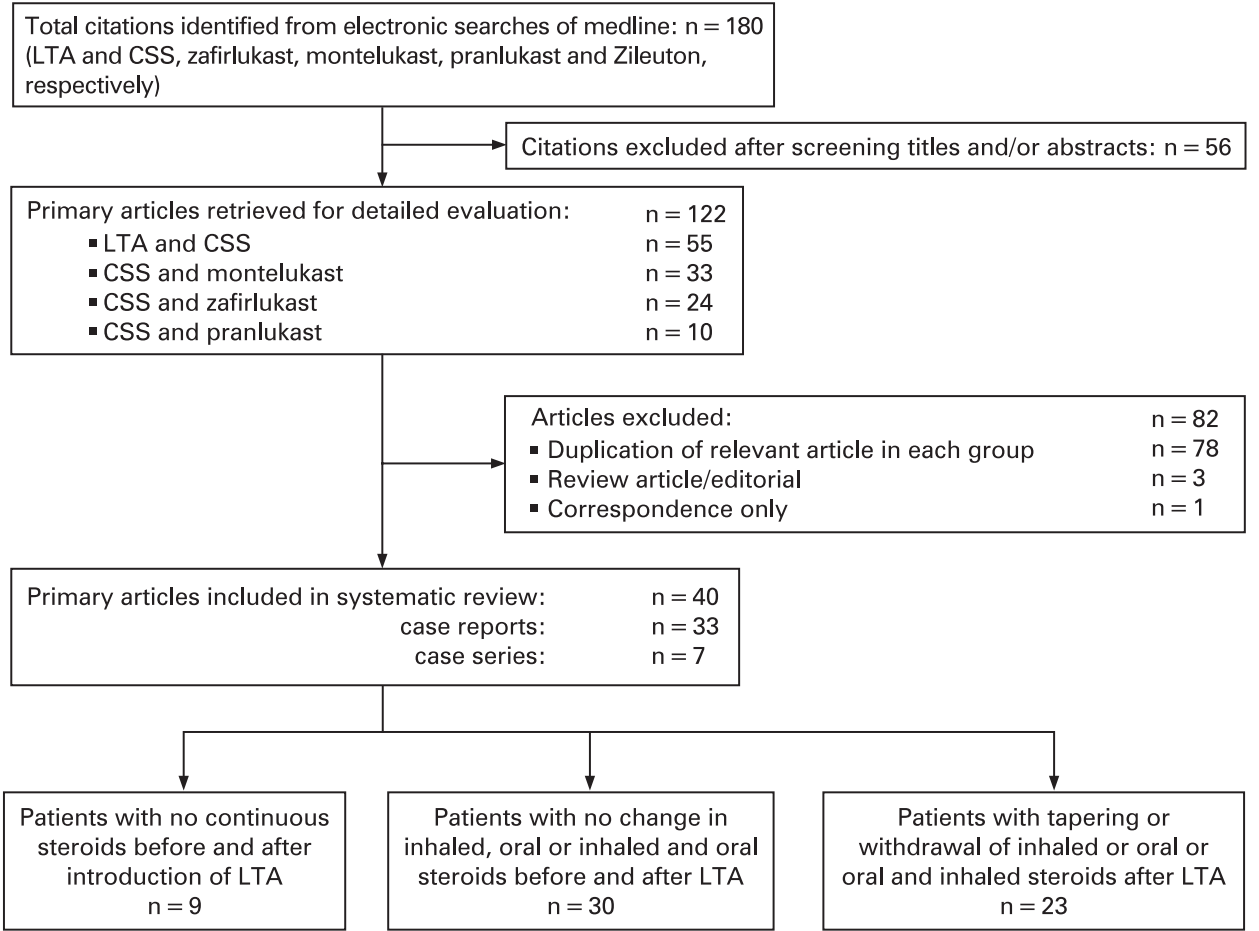

\section{Assessment of potential causal relationship between LTA use and CSS}

The cases were divided into three groups depending on steroid usage prior to LTA introduction (see box 1).

\section{Correct identification of the causative drug}

To our knowledge, we could not identify any additional drug that was started during the time period studied preceding the development of CSS in patients who received LTA.

Considering Hill's criteria of causation, clinical evidence of CSS should follow (not predate) the onset of treatment with the LTA.' Generally, in drug induced adverse reactions, this "latency period" may be as short as a few seconds (in patients with anaphylaxis or acute bronchospasm), a few hours (in transfusion related acute lung injury) or weeks to years in treatment with chemotherapeutic agents or radiation therapy. As such, there is no clear latency that proves or disproves drug causality.

\section{Statistical methods}

Organ involvement following exposure to each LTA was compared by cross tabulation using the $\chi^{2}$ test. The univariate

\section{Box 1}

- Group 1: 9 cases of Churg-Strauss syndrome post leukotriene antagonist (LTA), without antecedent continuous oral steroid therapy; 7 cases were entirely steroid naïve before initiation of LTA.

- Group 2: 30 cases - patients on stable doses of inhaled corticosteroids (ICS) $(n=27)$, ICS and oral corticosteroids (OCS) $(n=2)$ and OCS $(n=3)$ therapy only. Here LTA was added to improve asthma control.

- Group 3: 23 cases where LTA therapy led to improved control of symptoms and facilitated tapering or withdrawal of ICS $(n=1)$, OCS $(n=18)$ or OCS plus ICS $(n=2)$. odds ratio for each organ was calculated using logistic regression. The time from LTA exposure to CSS/number of organs involved with the Kruskal-Wallis test (with use of Dunn's multiple comparison post hoc test to examine the interval between LTA use and CSS onset in individual groups). Associations between ANCA status and LTA agent and organ involvement was compared by cross tabulation using the $\chi^{2}$ test. Univariate odds ratio for ANCA positive/negative was calculated using logistic regression.

To assess for independent predictors of organ pattern/ANCA status, we performed logistic regression analysis. To assess for independent predictors of the interval between LTA and CSS occurrence, we generated a general linear model. The predictor variables included in the multivariate model examining predictors of ANCA status were LTA type, group (1, 2 or 3) and number and pattern of organs involved (heart, lung, nerve, skin). The variables included when examining independent predictors of organ involvement were LTA type, group (1, 2 or 3 ) and ANCA status, with the fraction of cases with a given organ affected used as the dependent variable. Statistical analysis was performed using SPSS 9.0.

\section{RESULTS}

\section{Patient demographics}

Reports of 63 cases in which CSS followed LTA therapy were identified. Mean age at presentation was 47 (range 7-79) years and $33(55 \%)$ were female. Mean duration of asthma prior to presentation was 7.5 years (range 4 months to 20 years).

One reported case following Zileuton administration was excluded as it did not meet the ACR criteria for CSS. ${ }^{8}$ The remaining 62 cases had based their diagnosis on at least four out of six ACR criteria. Thirty-two of these included data on histological confirmation of diagnosis (nasal mucosa $n=1$, heart $n=4$, nerve $n=6$, skin $n=10$, lung $n=10$, liver $n=1$ ). There were no major variations in the diagnostic criteria used in the diagnosis of CSS for the majority of reported patients. 
Table 1 Association between LTA use and pattern of organ involvement

\begin{tabular}{|c|c|c|c|c|c|c|c|c|}
\hline \multirow[b]{2}{*}{ LTA type } & \multicolumn{2}{|l|}{ Lung } & \multicolumn{2}{|l|}{ Cardiac } & \multicolumn{2}{|l|}{ Nerve } & \multicolumn{2}{|l|}{ Skin } \\
\hline & p Value & OR $(95 \%$ Cl) & p Value & OR (95\% Cl) & p Value & OR $(95 \%$ Cl) & p Value & OR (95\% CI) \\
\hline \multicolumn{9}{|c|}{ Univariate analysis } \\
\hline Pranlukast & 0.14 & $0.2(0.02 \text { to } 1.8)^{*}$ & 0.006 & $0.15(0.03 \text { to } 0.6)^{*}$ & 0.09 & $2.9(0.8 \text { to } 10.6)^{*}$ & 0.71 & $0.8(0.2 \text { to } 2.6)^{*}$ \\
\hline Zafirlukast & 0.15 & $1.9(0.5 \text { to } 7.1)^{*}$ & 0.32 & $3.1(0.3 \text { to } 29.3)^{*}$ & 0.71 & $0.001\left(0 \text { to } 1 \times 10^{17}\right)^{*}$ & 0.28 & $2.1(0.5 \text { to } 8.1)^{*}$ \\
\hline Montelukast & 0.35 & 1.0 & 0.003 & 1.0 & 0.23 & 1.0 & 0.41 & 1.0 \\
\hline \multicolumn{9}{|c|}{ Multivariate analysis } \\
\hline Montelukast & 0.11 & 1.0 & 0.03 & 1.0 & 0.15 & 1.0 & 0.2 & 1.0 \\
\hline
\end{tabular}

We performed univariate and multivariate logistic regression examining predictors of the pattern of organ expression (dependent variable), the latter controlling for antineutrophil cytoplasmic antibody status and patient group.

${ }^{*}$ Compared with montelukast. LTA, leukotriene antagonist.

\section{Exclusion of other causes of drug induced CSS}

CSS associated with drugs other than LTA is a rare occurrence and only a few cases have been reported in the literature..$^{-15}$ Only one of our reported cases had been receiving a macrolide (rokitamicin) simultaneously. ${ }^{16}$ Thus we could not find significant evidence to suggest confounding associations with drugs previously reported to cause CSS.

\section{Organ involvement and LTA use}

Different LTAs implicated in the cases of CSS were montelukast (29 cases), zafirlukast (17 cases) and pranlukast (16 cases). The lung was the most common organ involved at presentation of CSS $(n=48)$ followed by nerve $(n=37)$ and skin $(n=24)$. Sixteen patients had cardiac involvement, eight of which belonged to a single series. ${ }^{17}$

We used univariate $\chi^{2}$ analysis to investigate the association between use of the three LTA agents and the occurrence of vasculitis in each of the organs (lung, heart, nerve and skin). The LTA used was significantly associated with the occurrence of cardiac disease $(p=0.001)$, but not with other organs (see supplementary table 2 online). Thus zafirlukast tended to be associated with lung and cardiac involvement whereas pranlukast tended to be associated with neurological CSS, but not cardiac disease, with montelukast associated with intermediate risk.

To assess for independent predictors of the pattern of organ involvement, we developed a multivariate logistic regression model for each organ using patient group (1,2 or 3), ANCA status and LTA type as predictor variables (table 1 ). Using this approach, there were no significant independent predictors of lung, nerve or skin involvement, whereas LTA type was independently associated with cardiac involvement $(p<0.05)$. These findings are summarised in fig 2.

\section{ANCA positivity and disease manifestation}

ANCA measurements were reported in 48 cases; it is unclear whether the remainder had ANCA measured and not reported or not measured at all. Of these 48 cases, 22 (46\%) were ANCA negative. Seventeen cases were p-ANCA positive, one was c-ANCA positive $(1: 640)^{18}$ and in eight the positive ANCA pattern was not specified. In 18 cases anti-myeloperoxidase ELISA was positive, in 22 it was negative and in 26 this was not reported.

ANCA positivity was associated with LTA type on univariate analysis, with a higher probability of this in those treated with
Figure 2 Pattern of organ involvement according to the leukotriene antagonist used. For each of the four organs (lung, cardiac, neurological and skin), the number of cases with involvement (black) is compared with the number without that particular organ involvement (white). ${ }^{*} \mathrm{p}<0.05$, as assessed by multivariate logistic regression analysis, controlling for patient group and antineutrophil cytoplasmic antibody status.
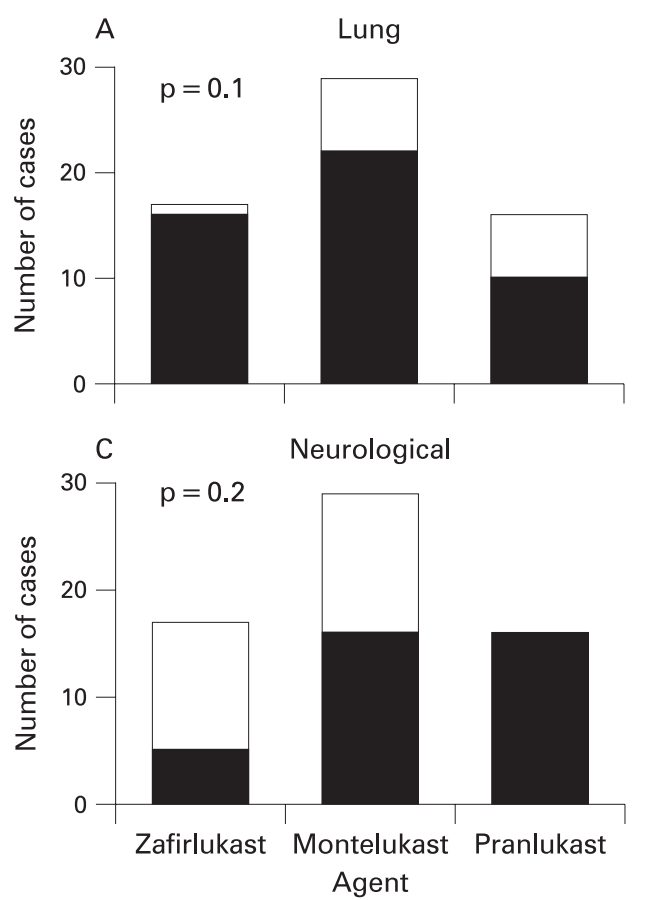
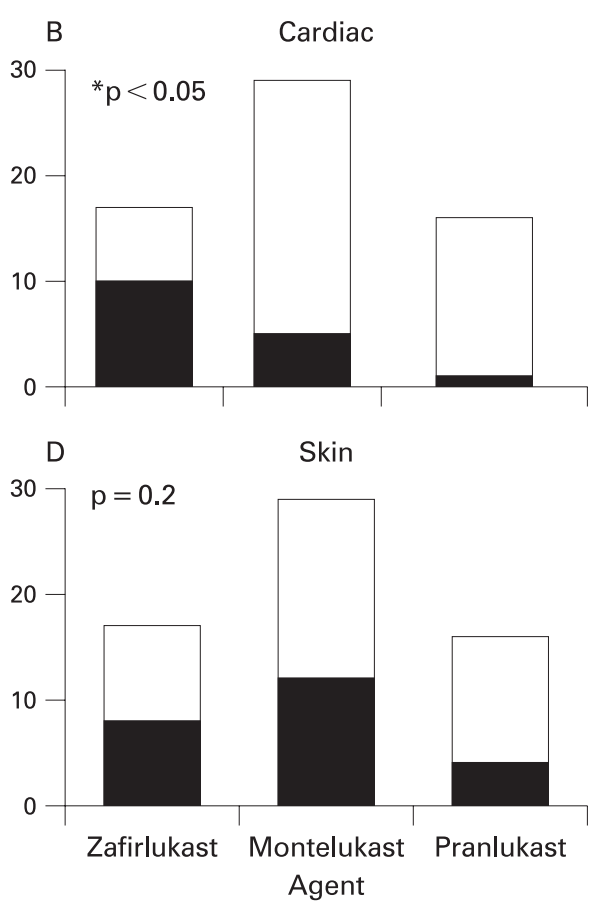
Table 2 Association between CSS phenotype and ANCA status (dependent variable)

\begin{tabular}{|c|c|c|c|c|c|c|c|}
\hline & $\begin{array}{l}\text { ANCA } \\
\text { positive } \\
(n=26)\end{array}$ & $\begin{array}{l}\text { ANCA } \\
\text { negative } \\
(\mathrm{n}=22)\end{array}$ & $\begin{array}{l}\text { ANCA } \\
\text { not } \\
\text { recorded }\end{array}$ & $\begin{array}{l}\text { p Value } \\
\text { (univariate } \\
\text { analysis) }\end{array}$ & $\begin{array}{l}\text { OR (logistic regression } \\
\text { analysis, } 95 \% \mathrm{Cl} \text { ) }\end{array}$ & $\begin{array}{l}\text { p Value } \\
\text { (multivariate } \\
\text { logistic } \\
\text { regression) }\end{array}$ & $\begin{array}{l}\text { OR (multivariate } \\
\text { logistic regression, } \\
95 \% \mathrm{Cl} \text { ) }\end{array}$ \\
\hline Pranlukast & 12 (80\%) & $3(20 \%)$ & 1 & $0.04 \dagger$ & $6.7(1.5 \text { to } 30)^{*}$ & 0.33 & $4.6(0.4 \text { to } 48)^{*}$ \\
\hline Zafirlukast & $5(56 \%)$ & $4(44 \%)$ & 8 & & $2.1(0.4 \text { to } 9.8)^{*}$ & 0.20 & $4.5(0.1 \text { to } 194)^{*}$ \\
\hline Montelukast & $9(38 \%)$ & $15(62 \%)$ & 5 & & 1.0 & 0.43 & 1.0 \\
\hline Heart & $3(38 \%)$ & $5(62 \%)$ & 8 & 0.3 & $0.4(0.09$ to 2.1$)$ & 0.29 & $0.12(0.003$ to 5.8$)$ \\
\hline Nerves & $19(68 \%)$ & $9(32 \%)$ & 9 & 0.02 & $3.9(1.1$ to 13.2$)$ & 0.19 & $8.6(0.3$ to 225$)$ \\
\hline Skin & $13(72 \%)$ & $5(28 \%)$ & 6 & 0.05 & $3.4(1.05$ to 12.0$)$ & 0.01 & $14.3(1.8$ to 110$)$ \\
\hline $\begin{array}{l}\text { Interval between LTA use } \\
\text { and CSS (mean (SEM)) }\end{array}$ & $6.7(2.3)$ & $5.9(1.0)$ & & 0.8 & & & \\
\hline $\begin{array}{l}\text { No of organs affected } \\
\text { (mean (SEM)) }\end{array}$ & $2.2(0.2)$ & $2.2(0.1)$ & & 0.95 & & 0.11 & \\
\hline
\end{tabular}

Univariate analysis was performed using the $\chi^{2}$ test with logistic regression (with a single predictor variable) used to generate ORs.

Using multivariate logistic regression analysis, lung involvement was independently associated with a negative ANCA test and skin involvement with a positive ANCA, but the association with LTA type and neurological involvement was lost.

* Relative to montelukast. †For all three drugs.

ANCA, antineutrophil cytoplasmic antibodies status; LTA, leukotriene antagonist; CSS, Churg-Strauss Syndrome.

pranlukast $(80 \%)$ and zafirlukast $(56 \%)(\mathrm{p}<0.05)$, and with advanced age (mean age 58 (range 21-79) vs 41 (range 1772) years; $p<0.05)$. The association of organ involvement with ANCA status is summarised in table 2.

Using multivariate logistic regression (table 2), ANCA positivity was independently associated with a low incidence of lung disease (odds ratio (OR) $0.1,95 \%$ confidence interval (CI) 0.01 to $0.98 ; \mathrm{p}<0.05)$ and a high incidence of skin involvement (OR 14.3, 95\% CI 1.8 to 110; $\mathrm{p}<0.05$ ), but not with the number of organs involved, the LTA used or the group. In addition, 12 of 22 (54\%) ANCA negative cases had evidence of vasculitis with extravascular eosinophilic infiltration on histological analysis. Thus ANCA status does not appear to influence eosinophilic infiltration.

\section{Influence of confounding drug therapy with oral or inhaled corticosteroids: groups 1, 2 and 3}

The characteristics of the patients in the different groups are outlined in supplementary table 3 (online).

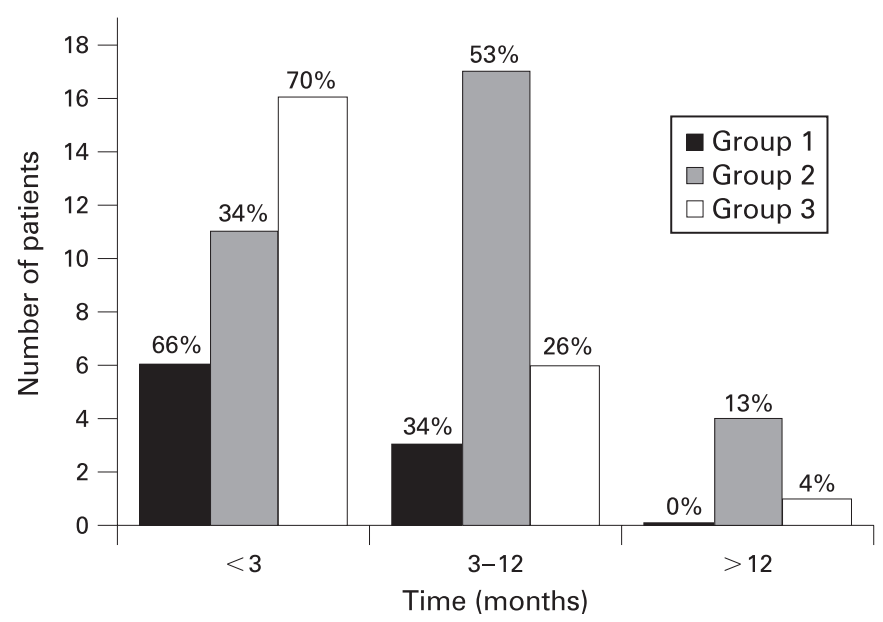

Figure 3 Interval between leukotriene antagonist use and onset of Churg-Strauss syndrome (CSS). The percentages refer to the fraction of each group developing CSS within the time scale specified.
Group 1 cases $(n=9)$ : no continuous OCS /ICS therapy when developed CSS

Seven cases were not receiving ICS or OCS when they started LTA. None of them met our criteria for suppressed or forme fruste disease.

Group 2 cases $(n=30)$ : patients on continuous ICS or OCS therapy prior to developing CSS

None of the group 2 cases had any alteration of either OCS or ICS therapy prior to developing CSS. Twenty-eight (93\%) of them developed CSS within 12 months of LTA therapy. It could be argued that the disease in these cases may have progressed with or without the introduction of LTA. It should be noted, however, that there was no change in steroid after LTA initiation.

Group $3(n=23)$ : patients started on LTA with subsequent reduction in OCS or ICS therapy.

In 23 cases, LTA led to better control of asthma symptoms thus facilitating tapering or withdrawal of ICS $(n=2)$, OCS $(n=19)$ and OCS plus ICS $(n=2)$, respectively. In these cases it is difficult to be sure that withdrawal of drug therapy did not bring about a relapse of a grumbling pre-existing CSS.

\section{Relationship between onset of CSS and starting LTA}

In our review, CSS was reported later than 12 months following LTA introduction in only 5 of 62 patients $(8.5 \%)^{19-23}$ : eight out of nine cases in group 1 (89\%), 22 out of 31 cases in group 2 $(71 \%)$ and 18 out of 22 cases in group $3(83 \%)$ presented within 6 months of initiating LTA therapy (fig 3)

\section{Recurrence of CSS on rechallenge with LTA}

Rechallenge followed by disease recurrence is an essential determinant of drug induced respiratory disease. Two cases suffered a relapse of CSS following reintroduction of LTA. One had been on LTA for 18 months before developing CSS. This was treated with OCS and withdrawal of LTA. Reintroduction of LTA caused a recurrence of blood eosinophilia and worsening symptoms despite a fixed continued dose of corticosteroids. ${ }^{24}$ In the second case, previously diagnosed CSS not related to LTA 
therapy had been controlled with OCS. A 1 week trial of montelukast resulted in relapse of CSS within 2 weeks. ${ }^{25}$

\section{Remission of signs and symptoms with removal of the drug}

In 52 of the 62 cases reported in this review (84\%), LTA therapy was stopped following development of CSS. Cessation of LTA was accompanied in most cases by escalation of treatment (summarised in supplementary table 4 online). In one case series, ${ }^{26} \mathrm{CSS}$ resolved following montelukast withdrawal despite no change in steroid dose. In the other two cases, montelukast was stopped and prednisolone dose was transiently increased, but was brought down rapidly within 1 week, suggesting that montelukast was the driving factor for ongoing vasculitis. In two other reported cases, disease manifestations resolved following stopping LTA therapy alone. These patients did not receive any steroid or other immunosuppressive therapy. ${ }^{8}{ }^{27}$ In 10 cases, it was not clear whether LTA therapy was stopped or not when CSS was treated.

\section{The presence of "form fruste" disease and influence of steroid withdrawal}

It could be argued that patients who develop CSS but had evidence of pre-existing disease were either in the final phase of developing CSS prior to adding LTA therapy or in a suppressed state where oral steroid withdrawal may have provoked progression to CSS. This pattern was identified in four patients $^{28-31}$ in groups $1-2(10 \%)$ and in seven (30\%) patients in group 3 , suggesting that in the majority of cases there was no evidence to substantiate steroid withdrawal as a trigger for the development of CSS.

\section{DISCUSSION}

The cases in this review cover a spectrum from patients with CSS without any preceding corticosteroid use, those where a suppressed CSS may have been precipitated by an abrupt withdrawal/tapering of the corticosteroids and patients with clear evidence of disease before the introduction of LTA (ie, those who may have progressed naturally). A significant number of patients who developed CSS did not have any suggestion of dormant disease or existing disease and showed a clear temporal relationship between LTA initiation and onset of CSS. In two cases, LTA treatment appears to have caused a relapse of CSS. ${ }^{24}{ }^{25}$ The published evidence in case series and reports therefore suggests an association between LTA and CSS that merits further consideration and may be causal.

The cases in this review cover a broad ethnic population and the full range of LTA available worldwide. There is a suggestion that zafirlukast might have a greater predilection for cardiac involvement, but we cannot exclude reporting bias because of the limited number of reports. Clearly, the manifestations of the disease might also be under immunological/pharmacogenetic influences. Several case control studies have suggested that CSS patients with ANCA positive disease differ from those without ANCA. Sinico et al found that ANCA positive cases had higher frequencies of renal involvement, alveolar haemorrhage, purpura and mononeuritis multiplex. ANCA negative cases had more cardiomyopathy and pulmonary infiltrates. In the French vasculitis study group, ANCA positive patients had greater renal involvement, sinusitis, neuropathy and purpura. ANCA positive patients also had greater evidence of vasculitic lesions on biopsy. In this context, it is interesting to note that there is an apparently equal distribution of organ involvement other than lung and skin involvement in both positive and negative ANCA cases in the reported drug induced/associated cases. ${ }^{4}$

Our group 1 patients, who were not on continuous steroid therapy, present the most convincing evidence of causation of CSS by LTA as none of these patients had any evidence of dormant or form fruste disease. A temporal relationship between initiation of LTA and development of CSS is apparent since more than half of the cases presented within 6 months of therapy.

Our group 2 patients, who had no alteration of any steroid therapy following introduction of LTA, represent the largest group of cases with CSS. Again, there was an apparent temporal relationship between the initiation of LTA therapy and the development of CSS within 12 months in 28 of 31 cases.

Our group 3 patients who had steroid therapy altered after the introduction of LTA represent cases where attributing their CSS to the initiation of LTA alone is difficult. Nevertheless, overall, this review suggests a temporal relationship of LTA therapy and CSS in 58 of 62 cases reported in the English literature.

This study has limitations. Although this review was limited to English language papers on the MEDLINE database, sufficient numbers of studies were available to perform meaningful data syntheses. It is also important to recognise that because of the publicity on a possible link between LTA exposure and CSS, most publications will have focussed on this association. It is interesting to note that associations have been reported for both omalizumab, high dose combination treatment with salmeterol and fluticasone, and disodium cromoglycate. ${ }^{32-34}$ We cannot therefore exclude the possibility of reporting bias in these publications. In view of the low incidence of CSS following treatment with LTAs, there are currently no controlled studies of sufficient power available to formally analyse the possible interaction between any drug exposure and CSS. As a result, our review was limited to case reports/series and, consequently, no formal assessment of the quality of evidence was possible.

Health care database analysis has been used to assess any relationships between LTA use and the development of CSS Using postmarketing surveillance, DuMouchel et al concluded that differences based on relative reporting were observed in the patterns of association of allergic granulomatous angitiis with LTA, ICS and $\beta_{2}$ agonist therapies. A strong association between LTA use and allergic granulomatous angitiis was present regardless of the use of other asthma drugs. ${ }^{35}$ Alternatively, a recent report utilising pooled data from two nested case control studies found no association between CSS and leukotriene modifiers after controlling for asthma drug use. ${ }^{36}$

A workshop in 2001 was sponsored by the National Heart, Lung and Blood Institute, to consider interrelationships among CSS, asthma and asthma therapeutics and to assess what is known about the underlying mechanisms of CSS. It concluded that treatment of patients with asthma with any of the leukotriene receptor antagonists, a 5-lipoxygenase inhibitor and ICS have been associated with CSS development but it was not known whether these agents were eliciting CSS. ${ }^{37}$

If LTA induce CSS, what is the mechanism? None of the case reports or case series have provided any clear mechanistic insight into the pathogenesis of LTA induced CSS. An imbalance in leukotriene receptor stimulation in patients with an underlying eosinophilic disorder has previously been suggested $^{38-40}$ and remains a potential cause of sustained eosinophilic stimulation which could promote the development of CSS. Unfortunately, there are few human data to suggest how 
LTA could induce CSS both with and without ANCA production or why ANCA status and organ involvement varies with the drug used. Clearly, further research focusing on the clinical phenotypic differences between ANCA positive and ANCA negative may provide insights into this dilemma.

\section{CONCLUSIONS}

Based on the available clinical evidence there is a suggestion of a causal relationship between LTA and CSS. Respiratory physicians need to be aware of the risk of CSS when treating patients with asthma with LTAs. Patients should be made aware of the signs and symptoms of CSS after initiation of LTA therapy and they should be regularly monitored for complications. The presence of more than two ACR criteria should make physicians explore the possibility of vasculitis before LTA therapy is introduced. ${ }^{28} 291$

Acknowledgements: University of Birmingham.

Funding: DRT is funded by a Wellcome research grant. MAL is funded by the HEFCE Senior Lecturer scheme. NN was funded by UHB Charities.

Competing interests: None.

\section{REFERENCES}

1. Watts RA, et al. Epidemiology of systemic vasculitis: a ten-year study in the United Kingdom. Arthritis Rheum 2000;43:414-19.

2. Masi AT, et al. The American College of Rheumatology 1990 criteria for the classification of Churg-Strauss syndrome (allergic granulomatosis and angiitis). Arthritis Rheum 1990;33:1094-100.

3. Lanham JG, et al. Systemic vasculitis with asthma and eosinophilia: a clinical approach to the Churg-Strauss syndrome. Medicine (Baltimore) 1984;63:65-81.

4. Sinico RA, et al. Prevalence and clinical significance of antineutrophil cytoplasmic antibodies in Churg-Strauss syndrome. Arthritis Rheum 2005;52:2926-35.

5. Sable-Fourtassou R, et al. Antineutrophil cytoplasmic antibodies and the ChurgStrauss syndrome. Ann Intern Med 2005:143:632-8.

6. Churg A, et al. Formes frustes of Churg-Strauss syndrome. Chest 1995;108:320-3

7. Hill AB. The Environment and Disease: Association or Causation? Proc R Soc Med 1965;58:295-300.

8. Dellaripa PF, et al. Recurrent panniculitis in a man with asthma receiving treatment with leukotriene-modifying agents. Mavo Clin Proc 2000;75:643-5.

9. Kjellbo H, Stakeberg H, Mellgren J. Possibly thiazide-induced renal necrotising vasculitis. Lancet 1965;14:1034-5.

10. Jarzobski J, et al. Vasculitis with allopurinol therapy. Am Heart J 1970;79:116-21.

11. Clarke BF, et al. Generalized hypersensitivity reaction and visceral arteritis with fatal outcome during glibenclamide therapy. Diabetes 1974;23:739-42.

12. Yermakov VM, Hitti IF, Sutton AL. Necrotizing vasculitis associated with diphenylhydantoin: two fatal cases. Hum Pathol 1983;14:182-4.

13. Gaffey CM, et al. Phenytoin-induced systemic granulomatous vasculitis. Arch Pathol Lab Med 1986;110:131-5.

14. Imai $\mathbf{H}$, et al. Carbamazepine-induced granulomatous necrotizing angiitis with acute renal failure. Nephron 1989:51:405-8.
15. Mathur S, Dooley J, Scheuer PJ. Quinine induced granulomatous hepatitis and vasculitis. BMJ 1990;300:613.

16. Richeldi $\mathbf{L}$, et al. Churg-Strauss syndrome in a case of asthma. Allergy 2002:57:647-8.

17. Wechsler ME, et al. Churg-Strauss syndrome in patients receiving montelukast as treatment for asthma. Chest 2000;117:708-13.

18. Gal AA, et al. Cutaneous lesions of Churg-Strauss syndrome associated with montelukast therapy. Br J Dermatol 2002;147:618-19.

19. Oberndorfer $\mathbf{S}$, et al. Churg Strauss syndrome during treatment of bronchial asthma with a leucotriene receptor antagonist presenting with polyneuropathy. Neurologia 2004;19:134-8.

20. Hashimoto $\mathbf{M}$, et al. Churg-Strauss syndrome after reduction of inhaled corticosteroid in a patient treated with pranlukast for asthma. Intern Med 2001:40:432-4.

21. Guilpain $\mathbf{P}$, et al. Churg-Strauss syndrome in two patients receiving montelukast. Rheumatology (Oxford) 2002;41:535-9.

22. Turvey SE, Vargas SO, Phipatanakul W. Churg-Strauss syndrome in a 7-year-old receiving montelukast and inhaled corticosteroids. Ann Allergy Asthma Immunol 2003;90:274.

23. Kawakami T, et al. Initial cutaneous manifestations consistent with mononeuropathy multiplex in Churg-Strauss syndrome. Arch Dermatol 2005:141:873-8.

24. Choi IS, et al. Churg-Strauss syndrome may be induced by leukotriene modifiers in severe asthma. Ann Allergy Asthma Immunol 2003;91:98.

25. Solans R, et al. Montelukast and Churg-Strauss syndrome. Thorax 2002;57:183-5.

26. Cuchacovich R, Justiniano M, Espinoza LR. Churg-Strauss syndrome associated with leukotriene receptor antagonists (LTRA). Clin Rheumatol 2007:26:1769-71.

27. Boccagni C, et al. Churg-Strauss syndrome associated with the leukotriene antagonist montelukast. Neurol Sci 2004;25:21-2.

28. Holloway J, et al. Churg-Strauss syndrome associated with zafirlukast. J Am Osteopath Assoc 1998;98:275-8.

29. Wechsler ME, et al. Pulmonary infiltrates, eosinophilia, and cardiomyopathy following corticosteroid withdrawal in patients with asthma receiving zafirlukast. JAMA 1998;279:455-7.

30. Tuggey JM, Hosker HS. Churg-Strauss syndrome associated with montelukast therapy. Thorax 2000;55:805-6.

31. Soy M, et al. Vasculitis induced by zafirlukast therapy. Clin Rheumatol 2002;21:328-9.

32. English J 3rd, et al. Fluticasone-associated cutaneous allergic granulomatous vasculitis. J Drugs Dermatol 2003;2:326-9.

33. Ruppert AM, et al. Development of Churg-Strauss syndrome with controlled asthma during omalizumab treatment. J Allergy Clin Immunol 2008;121:253-4.

34. Bili A, et al. Seven cases of complete and incomplete forms of Churg-Strauss syndrome not related to leukotriene receptor antagonists. J Allergy Clin Immuno 1999;104:1060-5.

35. DuMouchel W, et al. Association of asthma therapy and Churg-Strauss syndrome: an analysis of postmarketing surveillance data. Clin Ther 2004;26:1092-104.

36. Harrold LR, et al. Asthma drug use and the development of Churg-Strauss syndrome (CSS). Pharmacoepidemiol Drug Saf 2007:16:620-6.

37. Weller PF, et al. The relationship of asthma therapy and Churg-Strauss syndrome: NIH workshop summary report. J Allergy Clin Immunol 2001:108:175-83.

38. Franco J, Artes MJ. Pulmonary eosinophilia associated with montelukast. Thorax 1999:54:558-60.

39. Honsinger RW. Zafirlukast and Churg-Strauss syndrome. JAMA 1998;279:1949

40. Stirling RG, Chung KF. Leukotriene antagonists and Churg-Strauss syndrome: the smoking gun. Thorax 1999:54:865-6. 